\title{
Who benefits from a dietary online intervention? Evidence from Italy, Spain and Greece
}

\author{
Ralf Schwarzer $^{1, *}$, Lena Fleig ${ }^{1}$, Lisa M Warner ${ }^{1}$, Maryam Gholami ${ }^{1}$, \\ Lluis Serra-Majem ${ }^{2,3,4}$, Joy Ngo ${ }^{2}$, Blanca Roman-Viñas ${ }^{2,3}$, Lourdes Ribas-Barba ${ }^{2,3}$, \\ Alessandro Distante ${ }^{5}$, Evangelia Ntzani ${ }^{6,7}$, George Giannakis ${ }^{8}$ and Maria L Brandi ${ }^{9}$ \\ ${ }^{1}$ Freie Universität Berlin, Department of Psychology, Habelschwerdter Allee 45, D-14195 Berlin, Germany: \\ ${ }^{2}$ Nutrition Research Foundation, University of Barcelona Science Park, Barcelona, Spain: ${ }^{3} \mathrm{ClBERObn}$ \\ Physiopathology of Obesity and Nutrition, Institute of Health Carlos III, Madrid, Spain: ${ }^{4}$ Research Institute of \\ Biomedical and Health Sciences, University of Las Palmas de Gran Canaria, Las Palmas, Spain: ${ }^{5}$ Istituto Scientifico \\ Biomedico Euro Mediterraneo, Mesagne, Italy: ${ }^{6}$ Department of Hygiene and Epidemiology, University of loannina \\ School of Medicine, loannina, Greece: ${ }^{7}$ Center for Evidence-Based Medicine, Department of Health Services, Policy \\ and Practice, School of Public Health, Brown University, Providence, RI, USA: ${ }^{8}$ Hellenic Health Foundation, Athens, \\ Greece: ${ }^{9}$ Department of Surgery and Translational Medicine, University of Florence, Florence, Italy
}

Submitted 21 June 2016: Final revision received 9 September 2016: Accepted 14 September 2016: First published online 10 November 2016

\begin{abstract}
Objective: The traditional Mediterranean diet includes high consumption of fruits, vegetables, olive oil, legumes, cereals and nuts, moderate to high intake of fish and dairy products, and low consumption of meat products. Intervention effects to improve adoption of this diet may vary in terms of individuals' motivational or volitional prerequisites. In the context of a three-country research collaboration, intervention effects on these psychological constructs for increasing adoption of the Mediterranean diet were examined.

Design: An intervention was conducted to improve Mediterranean diet consumption with a two-month follow-up. Linear multiple-level models examined which psychological constructs (outcome expectancies, planning, action control and stage of change) were associated with changes in diet scores.

Setting: Web-based intervention in Italy, Spain and Greece.

Subjects: Adults ( $n$ 454; mean age 42.2 (SD 10.4) years, range 18-65 years; $n 112$ at follow-up).

Results: Analyses yielded an overall increase in the Mediterranean diet scores. Moreover, there were interactions between time and all four psychological constructs on these changes. Participants with lower levels of baseline outcome expectancies, planning, action control and stage of change were found to show steeper slopes, thus greater behavioural adoption, than those who started out with higher levels. Conclusions: The intervention produced overall improvements in Mediterranean diet consumption, with outcome expectancies, planning, action control and stage of change operating as moderators, indicating that those with lower motivational or volitional prerequisites gained more from the online intervention. Individual differences in participants' readiness for change need to be taken into account to gauge who would benefit most from the given treatment.
\end{abstract}

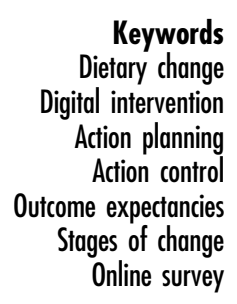

The traditional Mediterranean diet includes high consumption of fruits, vegetables, olive oil, legumes, cereals and nuts, moderate to high intake of fish, moderate intake of dairy products and low consumption of meat products. It has been found to protect overall health and is associated with a low risk of CVD ${ }^{(1-4)}$ and certain cancers ${ }^{(2,3,5-7)}$. Accordingly many institutions have recommended adopting and maintaining nutritional habits in line with this type of diet, and in particular with a focus on fruit and vegetable consumption. Although the WHO has advised to eat at least five portions of fruit and vegetables daily, globally fruit and vegetable consumption is lower than this recommendation ${ }^{(8,9)}$.

Thus, behavioural interventions are needed to promote the Mediterranean diet. Social-cognitive mechanisms of behaviour change can serve as a psychological underpinning of such interventions and a number of relevant 
intervention components have been identified ${ }^{(10)}$. Most of this research examines main effects of predictors such as attitudes, motivation, planning or social norms, as well as time by treatment interactions, to identify sources of outcome variation. This works well in randomized controlled trials but does not shed much light on behaviour change in single-arm interventions. Health promotion programmes that cannot, for whatever reason, include a control group usually examine pre-post differences that do not elucidate subgroup specificity of behaviour change. It is possible that psychological variables operate as moderators that might explain which study participants gain most from a treatment. This approach has been chosen in the present study. It was designed to test the feasibility of an online platform for lifestyle changes and to address possible moderation. A moderator is a third variable that affects the slope of the dependent on the independent variable. In other words, the moderator interacts with an independent variable. Putative moderators that might be responsible for differential treatment effects were explored. We briefly describe below four psychological constructs that may qualify as moderators when it comes to the prediction of dietary changes.

\section{Dietary stages of change}

Stage theories of behaviour change assume that one can categorize individuals in terms of their readiness for behaviour change by assigning them to stages that reflect a particular qualitative mindset. According to the Health Action Process Approach (HAPA) ${ }^{(11)}$, a three-stage distinction has been suggested, dividing study participants into pre-intenders who are unmotivated, intenders who are motivated but do not act accordingly, and actors who behave in line with their intentions. The likelihood for successful behaviour change is seen as dependent on the stage in which someone resides. This HAPA stage approach has been applied in the context of fruit and vegetable consumption ${ }^{(12)}$ but, to date, not to the Mediterranean diet (i.e. multiple dietary behaviours).

\section{Dietary outcome expectancies}

Outcome expectancies are beliefs about the consequences of one's action; such behavioural beliefs are regarded as being influential in developing a motivation to change one's health behaviours. People weigh the pros and cons of a certain behaviour, which means that they harbour either more positive or more negative outcome expectancies. Depending on this decisional balance they may develop an intention to act or an intention not to act. The term 'outcome expectancies' is used in SocialCognitive Theory ${ }^{(13)}$. The equivalent terms 'pros' and 'cons' are used in the Transtheoretical Model $^{(14)}$, where they represent the decisional balance when people contemplate whether to adopt a novel behaviour or not. In the Reasoned Action Approach ${ }^{(15)}$ the corresponding term is 'behavioural beliefs' that act as precursors of attitudes. In the context of weight management, for example, individuals may anticipate to feel more attractive as a result of dieting ${ }^{(16)}$. Similarly, consequences of adopting the Mediterranean diet could be imagined in terms of health benefits, fitness gains, weight control, or any social (e.g. my family appreciates healthy meals) or emotional (e.g. I feel good about my diet) outcome. Expecting such benefits of changing one's diet has repeatedly been shown to enhance the likelihood of dietary changes ${ }^{(10)}$.

\section{Dietary action planning}

Planning, as a self-regulation or self-management skill, is a proximal predictor for health behaviour change ${ }^{(17)}$. Action planning pertains to making detailed plans of when, where and how to initiate an action. It specifies a critical condition linked to goal-directed responses such as eating an adequate diet. Coping planning is supposed to be a more effective self-regulatory strategy than mere action planning. Only after people generate an action plan, they imagine possible barriers and generate coping strategies. Thus, coping planning comes on top of action planning, and their separate effects are often hard to disentangle (for a general review on planning health behaviours, see Hagger and Luszczynska $\left.{ }^{(18)}\right)$. Reviews on psychological intervention studies have documented the effects of planning on dietary behaviours $^{(19)}$.

\section{Dietary action control}

While planning is a prospective strategy (i.e. behavioural plans are made before the situation is encountered), action control is a concurrent self-regulatory strategy where the ongoing behaviour is continuously evaluated with regard to a behavioural standard. Action control can comprise three facets: self-monitoring ('I consistently monitored when, where and how I eat fruit and vegetables'), awareness of standards ('I have always been aware of my diet') and self-regulatory effort ('I took care to eat as I intended to') ${ }^{(20)}$. In intervention programmes, the selfmonitoring component is most frequently applied, for example by daily diary forms or calendars that are provided to allow for continuous self-monitoring during a treatment phase. The combination of planning and action control has been studied in the context of fruit and vegetable consumption $^{(12,21)}$.

\section{Aims of the current study \\ The present study aims to uncover the predictive role of HAPA stages of change, outcome expectancies, dietary planning and dietary action control when it comes to adopting or maintaining the Mediterranean diet. As it is a single-arm intervention, the question cannot be whether}


the treatment is superior to a control condition. Rather, the question is whether there are changes at all and whether the amount of change is related to any of the psychological constructs. Thus, it is expected that all four constructs (i.e. HAPA stages of change, outcome expectancies, planning and action control) are predictive of change, which means that higher prerequisites result in higher levels of the nutritional outcome measure. Moreover, the question is whether there are differential effects; that some participants change more than others. We hypothesize that those who enter the programme at a low level of psychological readiness have more to gain than those who enter at an already high level of nutritional awareness and motivation. Thus, the question is whether a moderator effect can be established, using the four psychological constructs as putative moderators in the analyses.

\section{Method}

\section{Participants and procedure}

The current pilot intervention study targeted adult residents in Italy, Greece and Spain in 2015 as part of Credits4Health ('Credits-based, people-centric approach for the adoption of healthy life-styles and balanced Mediterranean diet in the frame of social participation and innovation for health promotion', funded by the European Commission within the VII Framework Programme, Grant Agreement 602386). The Credits 4 Health $(\mathrm{C} 4 \mathrm{H})$ project uses an online platform with personalized motivational pathways with the aim to improve diet and activity levels in the Mediterranean countries. The purpose of the pilot study was to test the feasibility of an online platform designed for a future two-arm randomized controlled trial. Potential survey participants were recruited through radio messages, web announcements on the official project web page and local web pages, as well as through use of local mailing lists. We obtained ethical approval from all local review boards. Individuals were eligible to participate if they were apparently healthy, at least 18 years old and residing in one of the four selected areas in Italy, Spain and Greece. A total of 653 persons were screened and provided with information about the purpose of the study. After giving informed consent, they received an account for the online platform to complete a baseline questionnaire with behavioural and psychological items. Baseline nutrition data were available from 454 individuals (Italian, $n$ 155; Greek, $n$ 117; Spanish, $n$ 82) and 118 of them attended the follow-up assessment (26\%). Because six persons did not complete the diet index, the final longitudinal sample was 112 persons (forty-seven men, sixty-five women). Mean age was 42.2 (SD 10.36) years (range 18-65 years) and mean BMI was $26 \cdot 4$ (SD 5.2) kg/m (range $16.47-56.69 \mathrm{~kg} / \mathrm{m}^{2}$ ). Participants did not receive any incentives for their participation.

\section{Intervention}

The online platform delivered a lifestyle intervention that implemented theory-based behaviour change components. In the present paper we focus on the dietary components of the complex lifestyle intervention. Table 1 summarizes the applied behaviour change techniques, the according theoretical construct and implementation strategy.

\section{Measures}

\section{Dietary behaviours index}

Adherence to the Mediterranean diet was assessed with the Mediterranean Diet Adherence Screener (MEDAS), a validated fourteen-item index ${ }^{(22)}$. This screener is a heterogeneous nutritional habit index and scores were normally distributed in the current data set. Higher values on the MEDAS indicate a higher adherence to the Mediterranean dietary pattern. Some questions refer to food habits, such as 'Do you use olive oil as the principal source of fat for cooking?' Others ask about the frequency of consumption of certain foods, for example 'How many servings of vegetables do you consume per day?' and 'How many servings of fish/seafood do you consume per week?' Questions were scored as 0 or 1 according to the level of adherence to the Mediterranean diet recommendations.

\section{Psychological constructs}

Stages of change, outcome expectancies, planning and action control assessment were validated in previous studies $^{(20-24)}$ and the response format was from 1 ( = 'not at all true') to 6 ( $=$ 'completely true'). The inventories were administered in Italian, Spanish, Catalan and Greek languages. The MEDAS has also been pretested in the three countries along with the psychological variables ${ }^{(23)}$.

Positive diet-specific outcome expectancies. Positive diet-specific outcome expectancies were measured at baseline by three items, introduced by the stem 'If I eat five portions of fruit or vegetables a day ...' followed by statements regarding positive consequences, namely '... then I feel more satisfied and pleased', '... I feel more physically fit' and '... I look better'. Cronbach's $\alpha$ was 0.79 .

Diet-specific planning. Diet-specific planning was assessed at baseline by four items, two of them pertaining to action planning with the stem 'I have made a detailed plan for ...' followed by statements such as '... when, where, or how to eat fruit or vegetables' and '... how to maintain an overall balanced diet'. The other two items referred to coping planning with the response options: '.. which alternative diet I choose, in case I cannot adhere to my planned diet' and '... what to eat instead if I don't have time to shop or cook, or if any other interruption or obstacle emerges (e.g. eat with others in a restaurant)'. Cronbach's $\alpha$ was $0 \cdot 90$. 
Table 1 Intervention mapping of behaviour change techniques to theoretical constructs ${ }^{(33)}$ (numbers in CALO-RE taxonomy ${ }^{(34)}$ in square brackets, numbers in $\mathrm{v} 1$ taxonomy ${ }^{(35)}$ in parentheses)

\begin{tabular}{|c|c|c|}
\hline Theoretical construct & $\begin{array}{l}\text { Behaviour change technique } \\
\text { (number in v1 taxonomy) [number in } \\
\text { CALO-RE taxonomy] }\end{array}$ & Implementation strategy \\
\hline Risk perception & $\begin{array}{l}\text { Provide information on consequences } \\
\text { of behaviour to the individual [2] }\end{array}$ & $\begin{array}{l}\text { - Individualized risk feedback on self-reported abdominal girth (What } \\
\text { does your waistline ratio tell about you?) } \\
\text { - Individualized risk feedback on vessel calcination (How does a } \\
\text { Mediterranean diet affect your body?) }\end{array}$ \\
\hline \multirow[t]{2}{*}{ Outcome expectancies } & $\begin{array}{l}\text { Provide information on consequences } \\
\text { of behaviour in general [1] }\end{array}$ & $\begin{array}{l}\text { - Interactive quiz on nutritional facts and myths about nutritional } \\
\text { content of several foods (Sherlock Holmes Quiz) } \\
\text { - Interactive quiz on the Mediterranean pyramid (What do the } \\
\text { Egyptians have to do with nutrition?) }\end{array}$ \\
\hline & Pros and cons (9.2) & $\begin{array}{l}\text { - Open text fields to enter up to six advantages of engaging in a } \\
\text { Mediterranean diet for a comic character (Convince Sporty the frog } \\
\text { to eat more healthily) } \\
\text { - Open text fields to enter up to three short-term and three long-term } \\
\text { positive consequences of a Mediterranean diet for oneself } \\
\text { - Open text field to enter up to three disadvantages of a } \\
\text { Mediterranean diet for oneself } \\
\text { - Reappraisal of the disadvantages by generating positive outcomes } \\
\text { (preceded by three examples) }\end{array}$ \\
\hline \multirow[t]{3}{*}{ Self-efficacy } & Prompt self-talk (15.4) & - Open text field to enter a motivating sentence to eat more healthily \\
\hline & $\begin{array}{l}\text { Prompting focus on past success } \\
(15 \cdot 3)\end{array}$ & $\begin{array}{l}\text { - Introduction of three tempting situations, participants could choose } \\
\text { whether they had managed to resist these situations in the past } \\
\text { (Can I resist temptations?) } \\
\text { - Open text fields to document own nutritional success story (Are } \\
\text { you ready for the nutrition challenge?) }\end{array}$ \\
\hline & Vicarious reinforcement $(16 \cdot 3)$ & $\begin{array}{l}\text { - Provision of examples of success stories if participant did not recall } \\
\text { any mastery experience (tailored to gender of participant) }\end{array}$ \\
\hline \multirow[t]{2}{*}{ Planning } & Action planning (1.4) & $\begin{array}{l}\text { - Goal setting } \\
\text { - Weekly online planner } \\
\text { - Pop-up windows to specify nutritional component, number of } \\
\text { servings, location and occasion (e.g. after work) }\end{array}$ \\
\hline & Problem solving/coping planning (1.2) & $\begin{array}{l}\text { - Identification of potential barriers/challenging situations (self- } \\
\text { generated) and ways to overcome them (self-generated; if none } \\
\text { mentioned, examples provided) }\end{array}$ \\
\hline Action control & $\begin{array}{l}\text { Prompt self-monitoring of behaviour } \\
(2.3)\end{array}$ & $\begin{array}{l}\text { - Weekly review of online planner } \\
\text { - Slidebar to rate one's success }(0-100 \%) \text { of enacting action plans } \\
\text { - Enter alternative behaviours if action plans were not met }\end{array}$ \\
\hline
\end{tabular}

Diet-specific action control. Diet-specific action control was assessed at baseline with the following four statements: (i) 'I have been aware of my concrete dietary plan. It was always on my mind'; (ii) 'I have monitored how well I have adhered to my planned diet'; (iii) 'I have monitored how poorly I have adhered to my planned diet (e.g. snacking, indulging in tempting foods)'; and (iv) 'I have kept records regarding the amount of my fruit or vegetables'. Cronbach's $\alpha$ was 0.68.

Stages of change. Stage of change for adhering to the Mediterranean diet was assessed at baseline by a single question: 'Do you adhere to a healthy Mediterranean diet on a regular basis?' Participants were asked to choose one of the responses that ranged from 1 to $6(1=$ 'no, and I don't have this intention', 2 = 'no, but I am thinking about doing so', $3=$ 'no, but I am strongly committed to doing so', 4 = 'yes, but I feel it is too hard for me', $5=$ 'yes, and
I think I could manage to continue' and $6=$ 'yes, and it is easy for me'). Based on the responses, participants were then categorized into two groups based on their intention level, namely pre-intenders (those who do not have the intention to adhere to the target behaviour) and intenders/ actors (those who have the intention to engage in the behaviour but have not done yet and those who are already engaged in the health behaviour). Those who chose 1 or 2 were allocated to the category 'pre-intender'; those who chose 3, 4, 5 or 6, were included in the 'intender/actor' category. Validation of this assessment has been confirmed by Lippke et al. ${ }^{(25)}$.

\section{Covariates}

Sociodemographic data were included as covariates: sex, age and BMI $\left(\mathrm{BMI}=[\right.$ weight $(\mathrm{kg})] /\left[\right.$ height $\left.\left.(\mathrm{m})^{2}\right]\right)$ were recorded at the initial medical screening. 


\section{Data analysis}

Using the MIXED procedure in the statistical software package IBM SPSS Statistics Version 23.0, linear multilevel models were computed using age, BMI and sex as covariates, with two time points crossed in individuals (level 2) with REML (restricted maximum likelihood) estimation $^{(26,27)}$. We specified the time-varying MEDAS sum score as level-1 dependent variable. We studied cross-level interactions to determine the interrelationships between age (grand mean centred), BMI (grand mean centred), sex (coded as $1=$ men, $0=$ women) and time points (baseline $=0$, follow-up $=1$ ). The putative moderators were stage of change, outcome expectancies, planning and action control. In a linear mixed-effects model, the responses from participants are thought to be the sum of fixed and random effects. The fixed effects (model for the means) are of primary interest and random effects contribute to the covariance structure of the data. Adjustments for the covariance structure make the results more accurate. A series of analyses aimed at identifying a suitable model for the variances by comparing fit indices, mainly the Akaike's information criterion. An unstructured covariance matrix for random intercepts and random time effects was chosen. The covariance between intercept and linear growth parameter allows interpretation of differential effects such as whether the linear increase or decrease is related to initial values of a time-invariant predictor. As an effect size estimate, the total $R^{2}$ was computed which is the squared correlation between the actual outcome and the outcome predicted by the fixed effects.

\section{Results}

\section{Preliminary descriptives and dropout analyses}

The means, standard deviations and intercorrelations between the main study variables are presented in Table 2 . The mean MEDAS sum score was 7.44 (SD 1.34) at T1 (baseline) and 8.13 (SD 0.92) at T2 (follow-up). Men and women did not differ on any of the variables, and there were also no significant differences between the three countries.
A multivariate ANOVA with study dropout as the independent variable and the baseline assessments (age, BMI, sex, dietary behaviour, planning, outcome expectancies, action control and HAPA stage of change) as dependent variables revealed an overall statistically non-significant multivariate effect, Wilks' $\Lambda=0.96, \quad F(9, \quad 428)=1.86$, $P=0.06, \eta^{2}=0.04$. The result indicated that those who remained in the study at follow-up did not differ overall on baseline variables compared with those who dropped out (all variables, $P>0.05$, except action control, $P=0 \cdot 014$ ). Remainers scored lower (mean 2.04 (SD 0.91)) on action control than dropouts (mean 2.32 (SD 1.03)).

\section{Effects of stages of change on changes in dietary bebaviours}

The analyses aimed at testing the effects on dietary behaviour as measured by the MEDAS sum score. Linear mixed models were computed with time points nested in individuals, using MEDAS sum score at two time points as the level-1 dependent variable. Individual differences in terms of age, BMI and sex served as time-invariant covariates at level 2, and HAPA stage of change served as a predictor and putative moderator.

The findings (model for the means) are presented in Table 3 and Fig. 1. Time showed an increasing overall trend $(b=2 \cdot 04, P<0 \cdot 01)$. Sex $(P=0 \cdot 15)$ was not associated with the initial levels of MEDAS, but age $(b=0.02$, $P<0.01)$, BMI $(b=-0.04, P=<0.01)$ and stage of change ( $b=0.88, P=<0 \cdot 01$ ) were predictive, which means that somewhat older adults, those with lower BMI and with higher motivation (as reflected by stage) were more likely to have improved their dietary behaviours. These findings were qualified by a cross-level interaction between time and stage $(b=-0.79, P<0 \cdot 01)$ documenting a differential trajectory for initially less motivated and more motivated individuals. Total $R^{2}=0 \cdot 17$. Moreover, this was reflected in the model for the variances. The covariance between the intercept and the linear growth parameter was negative ( $\operatorname{cov}=-0 \cdot 90$, Wald $Z=-10 \cdot 68, P<0 \cdot 01)$. This suggests that pre-intenders had a steeper linear increase, whereas intenders/actors had a less steep increase in linear growth over time although they had overall better outcomes.

Table 2 Correlations, means and standard deviations, and pairwise mean comparisons (women $v$. men) among adults (mean age $42 \cdot 2$ (SD 10.4) years, range 18-65 years; $n 454$ at T1, $n 112$ at T2) participating in a web-based intervention in Italy, Spain and Greece, 2015

\begin{tabular}{|c|c|c|c|c|c|c|c|c|c|c|c|c|}
\hline & \multirow[b]{2}{*}{ Variable } & \multicolumn{5}{|c|}{ Correlation coefficients } & \multicolumn{2}{|c|}{ Men } & \multicolumn{2}{|c|}{ Women } & \multirow[b]{2}{*}{$F$} & \multirow[b]{2}{*}{$P$} \\
\hline & & 2 & 3 & 4 & 5 & 6 & Mean & SD & Mean & SD & & \\
\hline 1 & MEDAS T1 & $0.31^{\star *}$ & $0 \cdot 25^{\star \star}$ & 0.06 & $0 \cdot 25^{\star \star}$ & 0.13 & $7 \cdot 30$ & 1.43 & 7.54 & 1.26 & 0.89 & 0.35 \\
\hline 2 & MEDAS T2 & 1 & 0.02 & 0.03 & 0.03 & -0.02 & $8 \cdot 21$ & 0.98 & 8.03 & 0.88 & 1.06 & 0.31 \\
\hline 3 & Stage of change $\mathrm{T} 1$ & & 1 & $0 \cdot 37^{\star *}$ & $0 \cdot 31^{* *}$ & $0 \cdot 26^{\star \star}$ & 1.56 & 0.50 & 1.69 & 0.47 & 1.73 & 0.19 \\
\hline 4 & Outcome expectancies T1 & & & 1 & $0.34^{\star \star}$ & $0.44^{\star \star}$ & 3.67 & 1.21 & 3.77 & 1.21 & 0.20 & 0.66 \\
\hline 5 & Planning T1 & & & & 1 & $0 \cdot 70^{\star \star}$ & $2 \cdot 38$ & 1.36 & $2 \cdot 70$ & 1.27 & 1.63 & 0.21 \\
\hline 6 & Action control T1 & & & & & 1 & 2.08 & 0.95 & 1.97 & 0.89 & 0.34 & 0.56 \\
\hline
\end{tabular}

T1, baseline; T2, follow-up; MEDAS, Mediterranean Diet Adherence Screener

${ }^{* *} P<0.01$. For between-groups comparisons: $\mathrm{df}=1110$. 
Table 3 Results of linear mixed modelling: interaction of time and HAPA stage of change on healthy nutrition (MEDAS sum score) among adults (mean age 42.2 (SD 10.4) years, range 18-65 years; $n 454$ at T1, $n 112$ at T2) participating in a web-based intervention in Italy, Spain and Greece, 2015

\begin{tabular}{|c|c|c|c|c|c|c|c|}
\hline \multirow[b]{2}{*}{ Parameter } & \multirow[b]{2}{*}{ Estimate } & \multirow[b]{2}{*}{ SE } & \multirow[b]{2}{*}{ df } & \multirow[b]{2}{*}{$t$} & \multirow[b]{2}{*}{$P$} & \multicolumn{2}{|c|}{$95 \% \mathrm{Cl}$} \\
\hline & & & & & & Lower bound & Upper bound \\
\hline Intercept & $5 \cdot 81$ & 0.25 & $452 \cdot 02$ & 23.01 & $<0.01$ & $5 \cdot 32$ & $6 \cdot 31$ \\
\hline Time & 2.04 & 0.36 & $157 \cdot 25$ & 5.66 & $<0.01$ & 1.33 & $2 \cdot 75$ \\
\hline Sex & 0.17 & 0.12 & 337.88 & 1.43 & 0.15 & -0.06 & 0.39 \\
\hline Age & 0.02 & 0.01 & 354.63 & 4.39 & $<0.01$ & 0.01 & 0.03 \\
\hline BMI & -0.04 & 0.01 & 344.33 & -3.32 & $<0.01$ & -0.06 & -0.01 \\
\hline Stage & 0.88 & 0.14 & 438.90 & $6 \cdot 36$ & $<0.01$ & 0.61 & 1.16 \\
\hline Time $\times$ stage & -0.79 & 0.21 & 155.67 & -3.74 & $<0.01$ & -1.20 & -0.37 \\
\hline
\end{tabular}

HAPA, Health Action Process Approach; MEDAS, Mediterranean Diet Adherence Screener; T1, baseline; T2, follow-up.

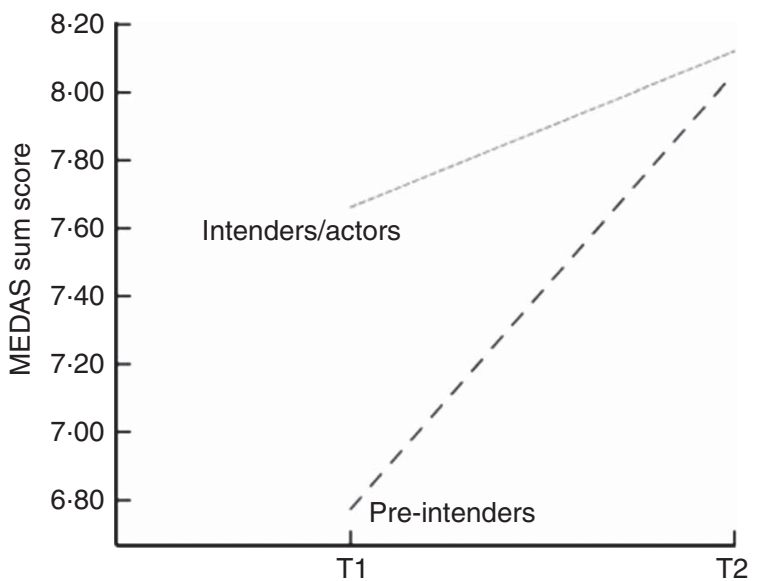

Fig. 1 Mean level changes in dietary behaviours (MEDAS sum scores) moderated by HAPA stages of change, controlling for age, sex and BMI, among adults (mean age 42.2 (SD 10.4) years, range 18-65 years; $n 454$ at $\mathrm{T} 1, n 112$ at T2) participating in a web-based intervention in Italy, Spain, and Greece, 2015. Less motivated persons have a steeper slope, which means that they gain more from the treatment (MEDAS, Mediterranean Diet Adherence Screener; HAPA, Health Action Process Approach; T1, baseline; T2, follow-up)

\section{Effects of positive outcome expectancies on changes in dietary behaviours}

The corresponding findings for positive outcome expectancies are presented in Table 4 and Fig. 2. Results for time, sex, age and BMI remained the same as before. Outcome expectancies $(b=0.24, P<0.01)$ were also predictive, which means that those participants with higher perceptions of the benefits of healthy eating were more likely to have improved their dietary behaviours. These findings were qualified by a cross-level interaction between time and outcome expectancies $(b=-0 \cdot 20$, $P=0.02$ ) documenting a differential trajectory for those with different levels of outcome expectancies. Total $R^{2}=0 \cdot 14$. Moreover, this was reflected in the model for the variances. The covariance between the intercept and the linear growth parameter was negative $(\operatorname{cov}=-0.94$, Wald $\mathrm{Z}=-10 \cdot 83, P<0 \cdot 01)$. This suggests that persons with less positive outcome expectancies had a steeper linear increase, whereas those with more positive outcome expectancies had a less steep increase in linear growth over time.

\section{Effects of dietary planning on changes in dietary behaviours}

The corresponding findings for dietary planning are presented in Table 5 and Fig. 3. Results for time, sex, age and BMI remained the same as before. Dietary planning ( $b=0.21, P<0 \cdot 01$ ) was significant. This means that those with higher levels of dietary planning were more likely to have improved their dietary behaviours. These findings were qualified by a cross-level interaction between time and planning $(b=-0.20, P=0 \cdot 01)$ documenting a differential trajectory for those with different levels of dietary planning. Total $R^{2}=0 \cdot 13$. Moreover, this was reflected in the model for the variances. The covariance between the intercept and the linear growth parameter was negative ( $\operatorname{cov}=-0.97$, Wald $Z=-11 \cdot 27, P=<0 \cdot 01)$. This suggests that persons with low levels of dietary planning had a steeper linear increase, whereas those with high dietary planning had a less steep increase in linear growth over time.

\section{Effects of dietary action control on changes in dietary behaviours}

The corresponding findings for dietary action control are presented in Table 6 and Fig. 4. Results for time, sex, age and BMI remained the same as before. Dietary action control $(b=0 \cdot 24, P<0 \cdot 01)$ was predictive, which means that those with higher levels of dietary action control were more likely to have improved their dietary behaviours. These findings were qualified by a cross-level interaction between time and dietary action control $(b=-0 \cdot 26$, $P=0.03$ ) documenting a differential trajectory for those with different levels of dietary action control. Total $R^{2}=0 \cdot 12$. Moreover, this was reflected in the model for the variances. The covariance between the intercept and the linear growth parameter was negative $(\operatorname{cov}=-1 \cdot 07$, 
Table 4 Results of linear mixed modelling: interaction of time and dietary outcome expectancies on healthy nutrition (MEDAS sum score) among adults (mean age 42.2 (SD 10.4) years, range 18-65 years; $n 454$ at T1, $n 112$ at T2) participating in a web-based intervention in Italy, Spain and Greece, 2015

\begin{tabular}{lccrrrrrr}
\hline & & & & & & \multicolumn{2}{c}{$95 \% \mathrm{Cl}$} \\
\cline { 3 - 8 } Parameter & Estimate & \multicolumn{1}{c}{ SE } & \multicolumn{1}{c}{ df } & \multicolumn{1}{c}{$t$} & $P$ & Lower bound & Upper bound \\
\hline Intercept & 6.37 & 0.22 & 453.75 & 28.33 & $<0.01$ & 5.93 & 6.81 \\
Time & 1.50 & 0.34 & 153.11 & 4.41 & $<0.01$ & 0.83 & 2.17 \\
Sex & 0.14 & 0.12 & 333.83 & 1.21 & 0.23 & -0.09 & 0.37 \\
Age & 0.02 & 0.01 & 343.00 & 4.53 & $<0.01$ & 0.01 & 0.04 \\
BMl & -0.04 & 0.01 & 342.49 & -3.53 & $<0.01$ & -0.06 & -0.02 \\
Expectancies & 0.24 & 0.05 & 439.44 & 4.60 & $<0.01$ & 0.14 & 0.34 \\
Timex expectancies & -0.20 & 0.09 & 15.04 & -2.33 & 0.02 & -0.37 & -0.03 \\
\hline
\end{tabular}

MEDAS, Mediterranean Diet Adherence Screener; T1, baseline; T2, follow-up.

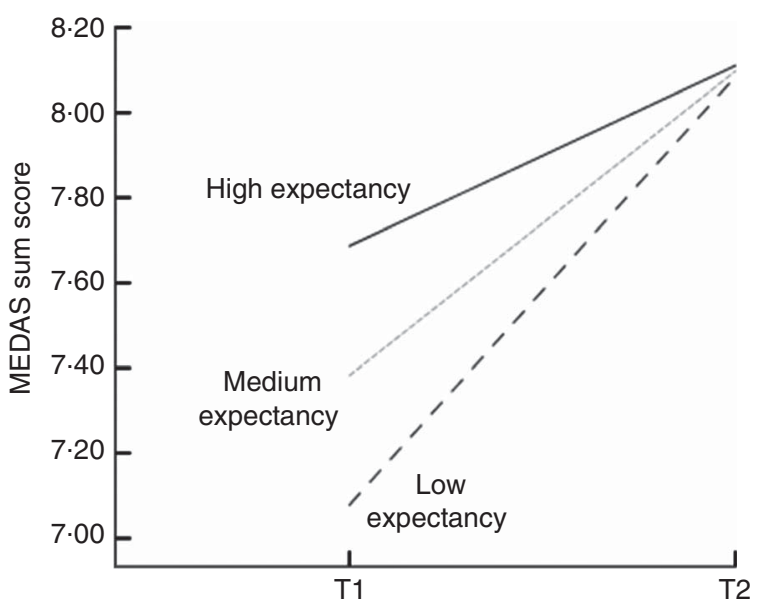

Fig. 2 Mean level changes in dietary behaviours (MEDAS sum scores) moderated by positive dietary outcome expectancies, controlling for age, sex and BMI, among adults (mean age 42.2 (SD 10.4) years, range $18-65$ years; $n 454$ at T1, $n 112$ at T2) participating in a web-based intervention in Italy, Spain and Greece, 2015. Persons who hold less positive outcome expectancies have a steeper slope, which means that they gain more from the treatment (MEDAS, Mediterranean Diet Adherence Screener; T1, baseline; T2, follow-up)

Wald $Z=-9.62, \quad P<0.01)$. This suggests that persons with low levels of dietary action control had a steeper linear increase, whereas those with high levels of dietary action control had a less steep increase in linear growth over time.

\section{Discussion}

The present study explored the possible psychological moderators that are involved in dietary change using data from a single-arm online intervention in three countries. There was an increase in self-reported healthy nutrition over time. Whereas previous research has focused on specific facets of nutrition (e.g. fruit and vegetable or fish consumption only ${ }^{(28)}$, the current theory-based behaviour change intervention proved to be feasible in promoting the Mediterranean diet, although its efficacy could not be fully evaluated due to the lack of a control group. As the main aim of the study, the role of four social-cognitive constructs was examined that were supposed to be related to successful behaviour change: HAPA stages of change, outcome expectancies, dietary planning and dietary action control, using age, sex and BMI as covariates. As expected, it turned out that higher scores on such baseline variables make it more likely that individuals improve their dietary behaviours. Outcome variance accounted for by the four constructs were given in terms of effect size estimates (total $R^{2}$ ), which equal $12 \%$ for action control, $14 \%$ for outcome expectancies, $13 \%$ for planning and $17 \%$ for HAPA stage of change. More importantly, there were differential effects of all four factors as they produced a similar pattern of interactions across all analyses. The lower people start when entering the programme, the steeper appears to be their increase in adopting a Mediterranean diet. At follow-up they come close or equal to those who entered at a higher level of readiness to change. Thus, the treatment has turned out to be an 'equalizer' helping those participants who were most in need for treatment. This is also reflected by the smaller variances at follow-up.

Previous research on dietary interventions has mainly focused on the evaluation of the treatment effect as reflected by the time by treatment interactions, and in longitudinal observation studies the focus has mainly been on the search for psychological predictors. The current approach has made an attempt to examine differential effects within a single-arm health promotion programme. This was done by identifying psychological moderators that may explain why some study participants gain more from a treatment than others. The results are in line with research on baseline by treatment interactions, although the term does not apply directly to the present design because it was a single-arm intervention. Such baseline by treatment interactions may indicate that some participants benefit from all interventions due to their pattern of study entry characteristics. For example, intenders as compared with actors showed larger increases in physical activity due to a planning intervention ${ }^{(29)}$. 
Table 5 Results of linear mixed modelling: interaction of time and dietary planning on healthy nutrition (MEDAS sum score) among adults (mean age 42.2 (sD 10.4) years, range 18-65 years; $n 454$ at T1, $n 112$ at T2) participating in a web-based intervention in Italy, Spain and Greece, 2015

\begin{tabular}{lcccrrrrr}
\hline & & & & & & \multicolumn{2}{c}{$95 \% \mathrm{Cl}$} \\
\cline { 3 - 8 } Parameter & Estimate & \multicolumn{1}{c}{$\mathrm{SE}$} & df & \multicolumn{1}{c}{$t$} & $P$ & Lower bound & Upper bound \\
\hline Intercept & 6.75 & 0.16 & 461.39 & 42.34 & $<0.01$ & 6.44 & 7.07 \\
Time & 1.27 & 0.22 & 158.10 & 5.65 & $<0.01$ & 0.82 & 1.71 \\
Sex & 0.14 & 0.12 & 327.34 & 1.18 & 0.24 & -0.09 & 0.37 \\
Age & 0.02 & 0.01 & 344.42 & 3.94 & $<0.01$ & 0.01 & 0.03 \\
BMl & -0.04 & 0.01 & 337.38 & -3.69 & $<0.01$ & -0.06 & -0.02 \\
Planning & 0.21 & 0.05 & 436.49 & 4.25 & $<0.01$ & 0.11 & 0.30 \\
Time & -0.20 & 0.08 & 154.66 & -2.64 & 0.01 & -0.36 & -0.05 \\
\hline
\end{tabular}

MEDAS, Mediterranean Diet Adherence Screener; T1, baseline; T2, follow-up.

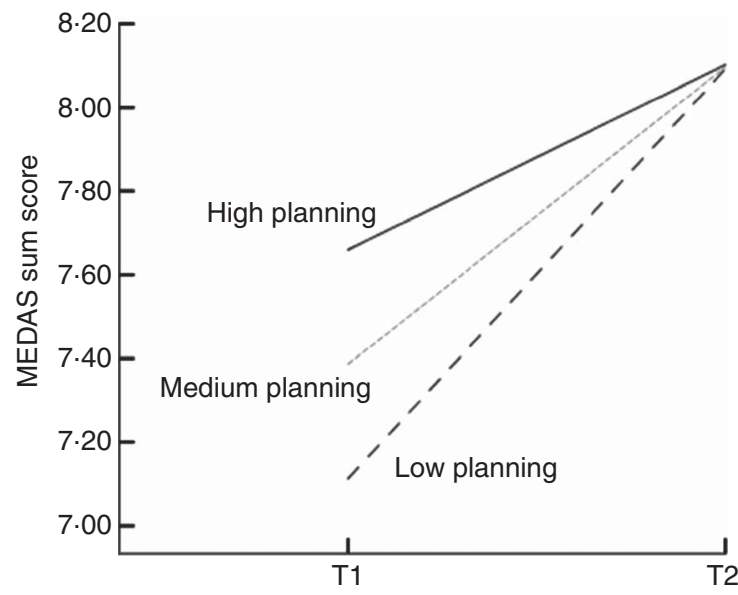

Fig. 3 Mean level changes in dietary behaviours (MEDAS sum scores) moderated by dietary planning, controlling for age, sex and BMI, among adults (mean age 42.2 (SD 10.4) years, range 18-65 years; $n 454$ at T1, $n 112$ at T2) participating in a webbased intervention in Italy, Spain and Greece, 2015. Persons who initially were less prone to plan their diet have a steeper slope, which means that they gain more from the treatment (MEDAS, Mediterranean Diet Adherence Screener; T1, baseline; T2, follow-up)

Some limitations need to be considered. First, the measurement of dietary consumption was self-reported which can cause bias as people may miss calculation of consumed portions in the previous weeks and may hold biased views about their own eating patterns. In spite of such potential bias, dietary self-reports have been validated against objective assessment such as biomarkers ${ }^{(30)}$. Second, there was an increase in self-reported healthy nutrition over time but the overall efficacy of the treatment could not be evaluated because this was not a randomized controlled trial, and no control group was available. Third, participants were self-selected and interacted with the platform on a self-selected frequency. Therefore, the results are not generalizable to other populations and one cannot determine a dose-response effect. Information on the frequency of logins and the amount of time spent on intervention pages was not available, which would have allowed us to determine dose-response effects.
Fourth, volunteers from three countries were included and these were not representative for the population of their countries of origin. Thus, no attempt was made to compare countries. It is likely that cultural eating habits make a difference and this should be studied on the basis of culture-representative samples.

Nevertheless, the present research contributes to the investigation of psychological entry characteristics that may allow a prediction of who would benefit most from this kind of dietary online intervention. Study participants were identified in terms of their HAPA stage of change, outcome expectancies, planning and action control, and all these variables taken together reflect a motivational mindset towards dietary change. Although overall the entire study sample made significant advances towards dietary change, these four psychological characteristics consistently moderated the amount of progress that individuals made. One cannot conclude that poorly motivated participants do always have a steeper increase but they did within the present treatment context. Thus, a future research question to be tackled is the type of treatment in terms of complexity, delivery mode (online, personal, group format, etc.) and selection of behaviour change techniques. One needs to find out whether there are aptitude-treatment interactions: individuals with characteristic A might benefit from treatment $X$, whereas those with characteristic B might benefit from treatment $\mathrm{Y}$.

To conclude, individual differences in participants' readiness for change and according psychological profiles need to be taken into account to gauge who would benefit most from the given dietary online treatment ${ }^{(31)}$. Findings from the present theory-based study have implications for future interventions to facilitate healthy eating behaviour. Diet-specific positive outcomes expectancies, planning and action control should be considered for inclusion in future intervention designs. These are alterable dietaryrelated variables ${ }^{(32)}$ and they are relatively easy to prompt in health behaviour change programmes. Finally, good practice for interventions and policies aiming at dietary behaviour should make use of behaviour change theory to optimize intervention outcomes and implementation ${ }^{(19)}$. 
Table 6 Results of linear mixed modelling: interaction of time and dietary action control on healthy nutrition (MEDAS sum score) among adults (mean age 42.2 (SD 10.4) years, range 18-65 years; $n 454$ at T1, $n 112$ at T2) participating in a web-based intervention in Italy, Spain and Greece, 2015

\begin{tabular}{lrrrrrrrr}
\hline & & & & & & \multicolumn{2}{c}{$95 \% \mathrm{Cl}$} \\
\cline { 3 - 8 } Parameter & Estimate & \multicolumn{1}{c}{$\mathrm{SE}$} & $\mathrm{df}$ & \multicolumn{1}{c}{$t$} & \multicolumn{1}{c}{$P$} & Lower bound & Upper bound \\
\hline Intercept & 6.80 & 0.18 & 333.01 & 38.49 & $<0.01$ & 6.46 & 7.15 \\
Time & 1.28 & 0.27 & 121.28 & 4.82 & $<0.01$ & 0.75 & 1.80 \\
Sex & 0.12 & 0.12 & 254.66 & 1.00 & 0.32 & -0.12 & 0.36 \\
Age & 0.02 & 0.01 & 262.04 & 3.98 & $<0.01$ & 0.01 & 0.03 \\
BMl & -0.04 & 0.01 & 257.55 & -3.70 & $<0.01$ & -0.07 & -0.02 \\
Action control & 0.24 & 0.07 & 318.51 & 3.45 & $<0.01$ & 0.10 & 0.37 \\
Time $\times$ action control & -0.26 & 0.12 & 116.46 & -2.22 & 0.03 & -0.50 & -0.03 \\
\hline
\end{tabular}

MEDAS, Mediterranean Diet Adherence Screener; T1, baseline; T2, follow-up.

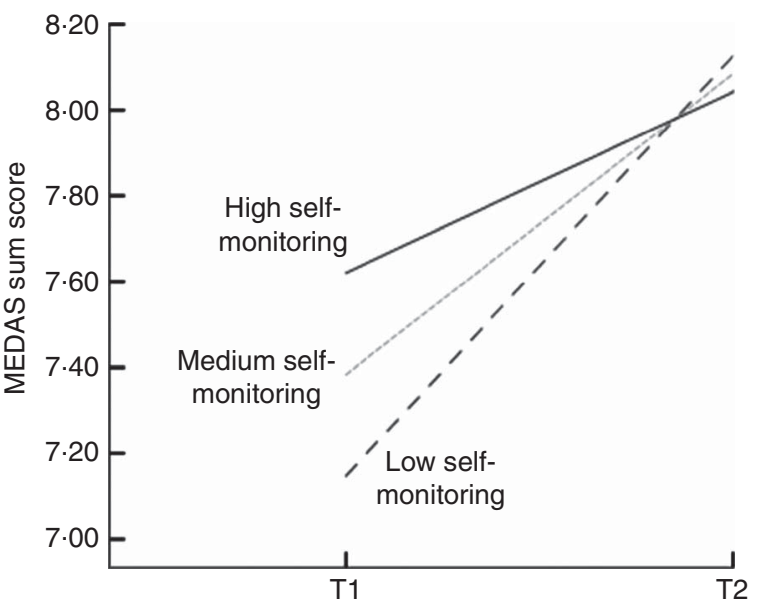

Fig. 4 Mean level changes in dietary behaviours (MEDAS sum scores) moderated by dietary action control, controlling for age, sex and BMI, among adults (mean age 42.2 (SD 10.4) years, range $18-65$ years; $n 454$ at T1, $n 112$ at T2) participating in a web-based intervention in Italy, Spain and Greece, 2015. Persons who initially were less aware of their dietary behaviour patterns have a steeper slope, which means that they gain more from the treatment (MEDAS, Mediterranean Diet Adherence Screener; T1, baseline; T2, follow-up)

\section{Acknowledgements}

Financial support: The project was funded by the European Commission within the VII Framework Programme FP7-HEALTH-2013-INNOVATION-1 - HEALTH.2013.3.3-1. Grant Agreement 602386: 'Credits-based, people-centric approach for the adoption of healthy life-styles and balanced Mediterranean diet in the frame of social participation and innovation for health promotion (Credits4Health)'. Conflict of interest: None. Authorship: Study design: M.L.B. Conceptualization of research questions: R.S. and L.F. Statistical methods: R.S. and L.M.W. Data analyses: R.S. and M.G. Preparation of manuscript: R.S., L.F., L.S.-M, J.N., B.R.-V., L.R.-B, A.D., E.N. and G.G. Ethics of buman subject participation: Ethical approval for the study was provided by Ethics Committee of Area Vasta Centro (Florence, Italy); Ethics Committee of ASL (Lecce,
Salento, Italy); Ethics Committee of ASL (Brindisi, Spain); Comisión de Bioética de la Universidad de Barcelona (Barcelona, Spain); and Greek National Ethical Committee (Greece).

\section{References}

1. Joshipura KJ, Hu FB, Manson JE et al. (2001) The effect of fruit and vegetable intake on risk for coronary heart disease. Ann Intern Med 134, 1106-1114.

2. Sofi F, Cesari F, Abbate R et al. (2008) Adherence to Mediterranean diet and health status: meta-analysis. BMJ 337, a1344.

3. Sofi F, Abbate R, Gensini GF et al. (2010) Accruing evidence on benefits of adherence to the Mediterranean diet on health: an updated systematic review and meta-analysis. Am J Clin Nutr 92, 1189-1196.

4. Estruch R, Ros E, Salas-Salvadó J et al. (2013) Primary prevention of cardiovascular disease with a Mediterranean diet. $N$ Engl J Med 368, 1279-1290.

5. Riboli E \& Norat T (2003) Epidemiologic evidence of the protective effect of fruit and vegetables on cancer risk. Am J Clin Nutr 78, 3 Suppl., 559S-569S.

6. Vainio H \& Weiderpass E (2006) Fruit and vegetables in cancer prevention. Nutr Cancer 54, 111-142.

7. Serra-Majem L, Roman B \& Estruch R (2006) Scientific evidence of interventions using the Mediterranean diet: a systematic review. Nutr Rev 64, 2 Pt 2, S27-S47.

8. Guilbert JJ (2003) The World Health Report 2002 - Reducing Risks, Promoting Healthy Life. Educ Health (Abingdon) 16, 230.

9. Hall JN, Moore S, Harper SB et al. (2009) Global variability in fruit and vegetable consumption. Am J Prev Med 36, 402-409.

10. Shaikh AR, Yaroch AL, Nebeling L et al. (2008) Psychosocial predictors of fruit and vegetable consumption in adults: a review of the literature. Am J Prev Med 34, 535-543.

11. Schwarzer R \& Luszczynska A (2015) Health action process approach. In Predicting Health Behaviours, 3rd ed., pp. 252278 [M Conner and P Norman, editors]. Maidenhead: McGrawHill Open University Press.

12. Godinho CA, Alvarez M-J, Lima ML et al. (2015) Health messages to promote fruit and vegetable consumption at different stages: a match-mismatch design. Psychol Health 30, 1410-1432.

13. Bandura A (1997) Self-Efficacy: The Exercise of Control. New York: Freeman.

14. Prochaska JO \& DiClemente CC (1983) Stages and processes of self-change of smoking: toward an integrative model of change. J Consult Clin Psychol 51, 390-395. 
15. Fishbein M \& Ajzen I (2010) Prediction and Change of Behavior: The Reasoned Action Approach. New York: Psychology Press.

16. Radtke T, Kaklamanou D, Scholz U et al. (2014) Are dietspecific compensatory health beliefs predictive of dieting intentions and behaviour? Appetite 76, 36-43.

17. Hagger MS, Luszczynska A, de Wit J et al. (2016) Implementation intention and planning interventions in health psychology: recommendations from the Synergy expert group for research and practice. Psychol Health 31, 814-839.

18. Hagger MS \& Luszczynska A (2014) Implementation intention and action planning interventions in health contexts: state of the research and proposals for the way forward. Appl Psychol Health Well Being 6, 1-47.

19. Adriaanse MA, Vinkers CD, De Ridder DT et al. (2011) Do implementation intentions help to eat a healthy diet? A systematic review and meta-analysis of the empirical evidence. Appetite 56, 183-193.

20. Sniehotta FF, Scholz U \& Schwarzer R (2005) Bridging the intention-behaviour gap: planning, self-efficacy, and action control in the adoption and maintenance of physical exercise. Psychol Health 20, 143-160.

21. Zhou G, Gan Y, Miao M et al. (2015) The role of action control and action planning on fruit and vegetable consumption. Appetite 91, 64-68.

22. Schröder H, Fitó M, Estruch R et al. (2011) A short screener is valid for assessing Mediterranean diet adherence among older Spanish men and women. J Nutr 141, 1140-1145.

23. Fleig L, Ngo J, Roman B et al. (2015) Beyond single behaviour theory: adding cross-behaviour cognitions to the health action process approach. BrJ Health Psychol 20, 824-841.

24. Kreausukon P, Gellert P, Lippke S et al. (2012) Planning and self-efficacy can increase fruit and vegetable consumption: a randomized controlled trial. J Behav Med 35, 443-451.

25. Lippke S, Ziegelmann JP, Schwarzer R et al. (2009) Validity of stage assessment in the adoption and maintenance of physical activity and fruit and vegetable consumption. Health Psychol 28, 183-193.
26. Heck RH, Thomas SL \& Tabata LN (2014) Multilevel and Longitudinal Modeling with IBM SPSS. New York: Routledge.

27. Hoffman L (2015) Longitudinal Analysis: Modeling WithinPerson Fluctuation and Change. Oxford: Routledge.

28. De Vet E, De Nooijer J, De Vries NK et al. (2006) The transtheoretical model for fruit, vegetable and fish consumption: associations between intakes, stages of change and stage transition determinants. Int J Behav Nutr Phys Act 3, 13.

29. Wiedemann AU, Lippke S, Reuter T et al. (2011) The more the better? The number of plans predicts health behaviour change. Appl Psychol 3, 87-106.

30. Steptoe A, Perkins-Porras L, McKay C et al. (2003) Psychological factors associated with fruit and vegetable intake and with biomarkers in adults from a low-income neighborhood. Health Psychol 22, 148-155.

31. Di Noia J, Contento IR \& Prochaska JO (2008) Computermediated intervention tailored on transtheoretical model stages and processes of change increases fruit and vegetable consumption among urban African-American adolescents. Am J Health Promot 22, 336-341.

32. Thomson CA \& Ravia J (2011) A systematic review of behavioral interventions to promote intake of fruit and vegetables. J Am Diet Assoc 111, 1523-1535.

33. Bartholomew LK, Parcel GS \& Kok G (1998) Intervention mapping: a process for developing theory and evidencebased health education programs. Health Educ Behav 25, 545-563.

34. Michie S, Ashford S, Sniehotta FF et al. (2011) A refined taxonomy of behaviour change techniques to help people change their physical activity and healthy eating behaviours: the CALO-RE taxonomy. Psychol Health 26, 1479-1498.

35. Michie S, Richardson M, Johnston M et al. (2013) The behavior change technique taxonomy (v1) of 93 hierarchically clustered techniques: building an international consensus for the reporting of behavior change interventions. Ann Behav Med 46, 81-95. 